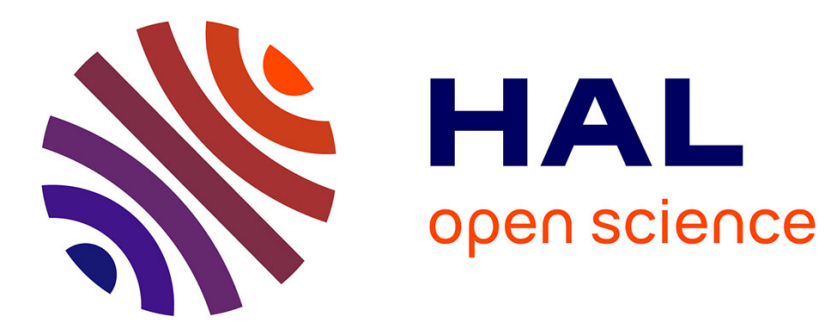

\title{
Comparing Network-Centric and Power Flow Models for the Optimal Allocation of Link Capacities in a Cascade-Resilient Power Transmission Network
}

Yi-Ping Fang, Nicola Pedroni, Enrico Zio

\section{- To cite this version:}

Yi-Ping Fang, Nicola Pedroni, Enrico Zio. Comparing Network-Centric and Power Flow Models for the Optimal Allocation of Link Capacities in a Cascade-Resilient Power Transmission Network. IEEE Systems Journal, 2014, PP (99), pp.1 - 12. 10.1109/JSYST.2014.2352152 . hal-01108169

HAL Id: hal-01108169

https://hal-centralesupelec.archives-ouvertes.fr/hal-01108169

Submitted on 22 Jan 2015

HAL is a multi-disciplinary open access archive for the deposit and dissemination of scientific research documents, whether they are published or not. The documents may come from teaching and research institutions in France or abroad, or from public or private research centers.
L'archive ouverte pluridisciplinaire HAL, est destinée au dépôt et à la diffusion de documents scientifiques de niveau recherche, publiés ou non, émanant des établissements d'enseignement et de recherche français ou étrangers, des laboratoires publics ou privés. 


\title{
Comparing Network-Centric and Power Flow Models for the Optimal Allocation of Link Capacities in a Cascade-Resilient Power Transmission Network
}

\author{
Y.-P. Fang, N. Pedroni, E. Zio, Senior Member, IEEE
}

\begin{abstract}
In this study, we tackle the problem of searching for the most favourable pattern of link capacities allocation that makes a power transmission network resilient to cascading failures with limited investment costs. This problem is formulated within a combinatorial multi-objective optimization framework and tackled by evolutionary algorithms. Two different models of increasing complexity are used to simulate cascading failures in a network and to quantify its resilience: a complex network model (namely, the Motter-Lai (ML) model) and a more detailed and computationally demanding power flow model (namely, the ORNL-Pserc-Alaska (OPA) model). Both models are tested and compared on a case study involving the $400 \mathrm{kV}$ French power transmission network. The results show that cascade-resilient networks tend to have a non-linear capacity-load relation: in particular, heavily loaded components have smaller unoccupied portions of capacity, whereas lightly loaded links present larger unoccupied portions of capacity (which is in contrast with the linear capacity-load relation hypothesized in previous works of literature). Most importantly, the optimal solutions obtained using the ML and OPA models exhibit consistent characteristics in terms of phrase transitions in the Pareto fronts and link capacity allocation patterns. These results provide incentive for the use of computationally-cheap network-centric models for the optimization of cascade-resilient power network systems, given the advantages of their simplicity and scalability.
\end{abstract}

Index Terms-power transmission network, cascading failures, complex network theory model, power flow model, capacity optimization, evolutionary algorithm

\section{INTRODUCTION}

$\mathrm{O}$ UR modern society has come to depend on large-scale critical infrastructures (CIs) to deliver resources and services to consumers and businesses in an efficient manner. These CIs are complex networks of interconnected functional and structural elements. Large scale outages on these real-world complex networks, although infrequent, are increasingly disastrous to our society, with estimates of direct

Y.-P. Fang is with the Chair on Systems Science and the Energetic challenge, École Centrale Paris and Supélec, Grande Voie des Vignes, 92290 Châtenay-Malabry, France (e-mail: yiping.fang@ecp.fr)

N. Pedroni is with the Chair on Systems Science and the Energetic challenge, École Centrale Paris and Supélec, Grande Voie des Vignes, 92290 Châtenay-Malabry, France (e-mail: nicola.pedroni@ecp.fr)

E. Zio is with the Chair on Systems Science and the Energetic challenge, École Centrale Paris and Supélec, Grande Voie des Vignes, 92290 Châtenay-Malabry, Paris, France and with Department of Energy, Politecnico di Milano, Milan, Italy (e-mail: enrico.zio@ecp.fr, enrico.zio@supelec.fr enrico.zio@polimi.it) costs up to billions of dollars and inestimable indirect costs. Typical examples include blackouts in power transmission networks [1]-[3], financial bankruptcy [4], telecommunication outages [5], and catastrophic failures in socio-economic systems [6], [7].

Research regarding modelling, prediction and mitigation of cascading failures in CIs, whereby small initial disturbances may propagate through the whole infrastructure system, has addressed the problem in different ways, including physical models for describing cascading failure phenomena [8]-[11], control and defense strategies against cascading failures [12]-[14], analytical calculation of capacity parameters [15], and modelling of the real-world data [16].

In particular, various problems concerning the robustness and functionality of CI systems (ranging from power outages and Internet congestion to affordability of public transportation) are ultimately determined by the extent to which the CI capability matches supply and demand under realistic conditions [17]. In this respect, the following two issues are closely related to each other and of significant interests: (i) how to improve the network resilience to cascading failures, and (ii) how to design CI systems with a reasonably limited cost. In most circumstances, high resilience and low cost are conflicting objectives and cannot be achieved simultaneously. For instance, a network whose components have high capacity can be highly resilient to failures; but, this type of components is often characterized by high costs.

Continuous effort has been made to model the capacity-load relationship of CI systems and to enhance the CI performance with limited cost. A homogeneous capacity-load relationship model has been widely used in the study of CIs [8], [9], [12]-[14], [18], whereby the capacity of a link (node) is assumed to be proportional to the initial flow of the link (node) (note that some of the studies focus on link modelling, while others concentrate on modelling node behaviour). However, it has been argued by Kim and Motter that this is unrealistic and empirical data suggests that the relationship between capacity and load of transmission lines is non-linear [17], [19]: heavily loaded lines usually have a lower tolerance parameter than lightly loaded lines. Most recently, Wang and Kim [20] proposed a (non-linear) two-step function for the relationship between the capacity and load of network vertices. Although based on an over-simplified model, it has been shown efficient to prevent cascades by protecting highest-load vertices. Li et al. [21] introduced a more complex heuristic capacity model whereby vertices with both higher loads and larger degrees are 
paid more extra capacities. It is shown that this model can achieve better network robustness than previous models under the same amount of available resources.

In the present study, we tackle the issue from a systematic perspective by searching for the strategy of resource (capacity) allocation in a power transmission network that is most favourable for resisting to cascading failures, while keeping the total resource (capacity) limited (i.e., while minimizing the network cost). This serves as the primary objective of this paper. In more detail, the problem is formulated within a large-scale, nonlinear and combinatorial multi-objective optimization framework and is solved by a fast and elitist genetic algorithm, namely NSGA-II [22].

The search by the NSGA-II requires also: (i) the construction of a model to describe the cascading failure process in the network of interest, and (ii) the repeated evaluation of the model for every possible capacity allocation pattern proposed by the algorithm during the search. With respect to the model, two approaches are typically considered in the analysis of power transmission systems: complex network theory models, such as the Motter-Lai (ML) model [8], [9] and artificial power flow models, such as the ORNL-Pserc-Alaska (OPA) model [10], [11], [39]. These approaches provide different tradeoffs between the (relatively low) computational cost associated to the model evaluation (allowing applications to large scale power grids) and the (high) level of detail in the system description (including physical characteristics and power flows constraints), respectively.

The OPA model seeks to faithfully describe the dispatching dynamics of the power flows during the evolution of the failure propagation following the initial disturbances, by explicitly incorporating the standard DC power flow equations and minimizing generation cost and load shedding [10]. Embracing this more physical description and solving the constrained linear optimization functions associated to the model, results in a significant increase in the computational burden, rendering practical application extremely difficult for realistic networks with large numbers of elements [23]. For these reasons, topological models based on complex network theory (e.g. the ML model) have emerged in recent years [8], [9], [13], [14], [18], [24]-[26]. In particular, the ML model is a relatively simple and abstract model relying on the resemblance of complex networks to electrical infrastructure systems (in terms of graph theory). It has the advantage of modelling cascading dynamics with few parameters, so that its application to realistic, large-scale networks is feasible and certainly more readily than OPA [16]. However, ML abstracts the power flow laws and constraints of the electrical system. Inevitably, then, it cannot provide direct physical measures of blackout size, but rather abstract measures such as efficiency loss. This has posed questions on whether or not it is adequate in practice, due to its abstract nature, although it has been recognized to offer a new and interesting perspective on the study of cascading failures on power grids [23].

It is worth mentioning that studies tackling the problem of comparison between network-centric approaches and power flow approaches are few in literature. Some studies [23], [25],
[27] have provided qualitative comparisons between complex network theory models and power flow models - identifying similarities and differences, and evaluating advantages and disadvantages. Most recently, Correa and Yusta conclude on the appropriateness of graph theory techniques for the assessment of electric network vulnerability by comparison to physical power flow models [28]. By extensive comparative simulation, Cupac et al. have shown that a network-centric model (CLM) exhibits ensemble properties which are consistent with the more realistic OPA fast-scale model [29]. Along these lines, our study takes the comparison a step forward by analyzing the optimization results, enabling to find more interesting insights.

In the present paper, we embrace both the ML and OPA cascading failure models and embed them within NSGA-II for optimally solving the problem of capacity resource allocation. With respect to that, the second objective of the paper is to study the possibility of using a simplified network-centric model (instead of a detailed power flow model) within an optimization framework, without affecting the quality of the optimal solutions found. For illustration, we apply the method to the $400 \mathrm{kV}$ French power transmission network, under the objectives of maximizing network resilience to cascading failures and minimizing investment costs. Finally, we systematically compare the results obtained by using the two cascading failure models of different complexity.

The reminder of this paper is organized as follows. In Section II, we introduce the ML and OPA cascading failure models in detail. We, then, formulate the multi-objective optimization problem taking investment costs and failure resilience into account in Section III. In Section IV, we briefly introduce the procedure of the NSGA-II algorithm. Section V illustrates the French $400 \mathrm{kV}$ power transmission network case study and the analysis and comparison of the results. Discussion and conclusion are given in Section VI.

\section{Models Of CASCADING FAILURe CONSIDERED IN ThIS WORK}

Modelling the dynamic evolution of system-wide cascading failure processes poses a number of challenges due to the diversity of mechanisms which can trigger the initial failure and influence the subsequent propagation of breakdowns in the power system [27]. Various cascading failure models have been proposed; these can be divided into two main categories: those based on complex network theory analysis and those using power flow analysis, often including optimal economic power dispatch after each failure in the propagation, e.g., by linear optimal power flow (OPF) [29].

Complex network theory models, including the ML model adopted in this work and described in Section A below, abstract the representation of a power grid as a graph and then study the connectivity characteristics, the propagation mechanisms through the graph connections and their relationships. These types of models have proved to provide a good understanding of the specific grid dynamics of cascading failures [30]. However, in these models the assumptions only abstract the real loading of the components and the flow distribution 
through the connections. For this reason, it is necessary to ascertain the meaningfulness of the results for real electrical infrastructures.

Power flow models, on the contrary, are based on realistic power flow equations to describe the flow dispatching dynamics and failure evolution after the initial disturbances in the power grid. The OPA model, which is the most commonly used of this type of models, is introduced in Section B below and is based on the DC power flow approximation [31].

\section{A. The ML Model}

The original ML model has been proposed by Motter and Lai [8], with extensions to differentiate generators and loads [16]. Here, the extended ML model in terms of transmission line failures is utilized. The power transmission network is represented as an undirected graph $Q$ with a set of $N$ vertices representing $N_{G}$ generators and $N_{D}$ loads representing distribution substations, interconnected by a set of $M$ edges representing transmission lines. The structure of the network is identified by an $N \times N$ interaction matrix $W$, whose element $w_{i j}$ is 0 if node $i$ and $j$ are not connected directly; otherwise it is assigned a value of 1 , for an unweighted network, or another numerical value, for a weighted network (as in the case of the work in the present paper).

The ML model assumes that at each time step, one unit of the relevant quantity (e.g., electrical flow for power grids) is exchanged between every pair of generator and distributor nodes, and transmitted along the shortest path connecting them. Then, the flow at one link is computed as the number of shortest paths passing through it. More precisely, the flow $F_{l}^{M L}$ of link $l$ is quantified by the link betweenness, calculated as the fraction of the generator-distributor shortest paths passing through that link:

$$
F_{l}^{M L}=\frac{1}{N_{G} N_{D}} \sum_{i \in V_{G}, j \in V_{D}} \frac{n_{i j}(l)}{n_{i j}}, l \in E
$$

where $E(\|E\|=M)$ is the set of all the links in the network; $V_{G}$ $\left(\left\|V_{G}\right\|=N_{G}\right)$ and $V_{D}\left(\left\|V_{D}\right\|=N_{D}\right)$ are the sets of generators and distributors, respectively; $n_{i j}$ is the number of shortest paths between generator nodes and distributor nodes, and $n_{i j}(l)$ is the number of generator-distributor shortest paths passing though link $l$.

In the original ML model [8], a homogeneous capacity-load relationship is assumed: the capacity of link $l$ is assumed to be proportional to its initial flow $F_{l}^{M L}(0)$ with a network tolerance parameter $\alpha$ :

$$
C_{l}^{M L}=(1+\alpha) F_{l}^{M L}(0), l \in E
$$

The concept of tolerance parameter $\alpha(\alpha \geq 0)$ can be understood as an operating margin allowing safe operation of the component under potential load increment ${ }^{1}$. The occurrence of a cascading failure is initiated by removal of a link, which in general changes the distribution of shortest paths. Then, the

\footnotetext{
${ }^{1}$ In this paper, the link capacities are variables to be optimized (see Section III); thus, assumption (2) is obviously not introduced in the problem formulation of the present work.
}

flow at a particular link can change and if it increases and exceeds its capacity, the corresponding link fails. Any failure leads to a new redistribution of loads and, as a result, subsequent failures can occur.

Using this cascading failure model, the damage of the network $Q$ can be characterized by the fraction of network efficiency lost in the cascading failure:

$$
V_{M L}=\frac{E(Q)-\overline{E(Q)}}{E(Q)}
$$

where $V_{M L} \in[0,1]$ and $\overline{E(Q)}$ represents the residual network structure after the cascading failure. $E(Q)$ measures the network efficiency based on the node pair shortest path distance between generators and distributors. For its computation all pairs of nodes $i \in V_{G}$, and $j \in V_{D}$ are weighted by the inverse of their distance:

$$
E(Q)=\frac{1}{N_{G} N_{D}} \sum_{i \in V_{G}} \sum_{j \in V_{D}} \frac{1}{d(i, j)}
$$

where $d(i, j)$ is the number of edges for an unweighted network or the sum of edge weights for a weighted network in the shortest path from $i$ to $j$ (like in the present case).

The geodesic network damage $V_{M L}$ measures the functionality of a network when subjected to a contingency due to cascading link disruption with regard to its steady state (base case). As $V_{M L}$ increases, the impact on the network due to cascading failure also increases, as some components become disrupted. $V_{M L}$ has proved to be a well-defined index being capable of providing results consistent with those of physical model indices [28].

The detailed simulation of the ML cascading failure model proceeds as follows:

(1) A random link is chosen as failed and, thus, is removed from the network.

(2) Recur to Eq. (1) and Floyd's shortest paths algorithm to calculate the flow of each working link in the network [32].

(3) Test each link for failure: for each link $l \in E$ of the network, if $F_{l}^{M L}>C_{l}^{M L}$ then link $l$ is regarded as failed and, thus, is removed from the network.

(4) If any working link fails, return back to step 2. Otherwise, terminate the simulation and evaluate the network damage by Eq. (3).

Complex network theory models, such as the ML that we use within our optimization framework of the following Section III, have no direct physical relation to the mechanisms of realistic power grids, but they have the key advantage that by utilizing techniques from graph theory they can be applied to analyze large-scale networks. For this reason, this modelling approach is seeing increasing applications for modelling cascading failure processes in power grids.

\section{B. The OPA Model}

The OPA model has been proposed by researchers at Oak Ridge National Laboratory (ORNL), Power System Engineering Research Center of Wisconsin University (PSerc), and Alaska University (Alaska) [10], [11]. The OPA model is 
built upon the Self-Organized Criticality (SOC) theory, contains two different time scale dynamics, i.e., fast power flow dispatching dynamics and slow power grid growth dynamics, and describes the complexity and criticality of power systems. It is a novel and powerful tool for analysing power systems. Our analysis focuses on the fast power flow dynamics, in order to ensure comparability with the ML model shortest path assumption.

The cascading failure model is based on the standard DC power flow equation,

$$
F^{O P A}=A \cdot P
$$

where $F^{O P A}$ is a vector whose $M$ components are the power flows through the lines, $F_{l}^{O P A}(l \in E), P$ is a vector whose $N-1$ components are the power injection of each node, $P_{i}(N$ is the total number of nodes in the network), with the exception of the reference generator, $P_{0}$, and $A$ is a constant matrix that depends on the network structure and impedances (see Ref. [10] for details about the computation of $A$ ). The reference generator power is not included in the vector $P$ to avoid singularity of $A$ as a consequence of the overall power balance.

The generator power dispatch is solved using standard linear programming methods. Using the input power demand, the power flow Eq. (5) is solved with the condition of minimizing the following cost function:

$$
f=\sum_{i \in V_{G}} P_{i}(t)+K \sum_{j \in V_{D}} P_{j}(t)
$$

This definition gives preference to generation shift whilst assigning a high cost (set $K=100$ ) to load shedding, and it is assumed that all generators operate at the same cost and that all loads are served with equal priority. The minimization is done with the following constraints:

(5) Generator power injections are generally positive and limited by installed capacity limits: $0 \leq P_{i} \leq$ $P_{i}^{\max }, i \in V_{G}$.

(6) Loads always have negative power injections: $P_{j}^{\text {dem }} \leq P_{j} \leq 0, j \in V_{D}$.

(7) The flow through links is limited by link capacities: $\left|F_{l}^{O P A}\right| \leq C_{l}^{O P A}$.

(8) Total power generation and consumption remain balanced: $\sum_{i \in V_{G} \cup V_{D}} P_{i}=0$.

Notice that in order to simplify the power flow problem, making it linear, a number of assumptions have been made in the standard formulation of DC power flow, one of which is that the transmission line resistance is assumed to be negligible i.e. $\mathrm{R}<<\mathrm{X}$, i.e. lines are assumed without loss [31]. This means that the loss of power transmission is neglected in the original OPA cascading failure model [10]. However, the objective of cost minimization (Eq. (6)) is only applied to guide the generator power redispatch after the occurrence of a transmission line failure, for which changes in generation or load shedding are usually considered, as the change in transmission loss among different redispatch strategies should probably not be large and considered by the network operator [10].
After solving the linear optimization by using the simplex method as implemented in Flannery et al. [33], we examine which lines are overloaded. A line is considered to be overloaded if the power flow through it is within $1 \%$ of the limit capacity $C_{l}^{O P A}$. Each overloaded line may outage with probability $p_{1}$ ( $p_{1}$ is set as 1 in the case study to ensure its comparability with ML). If an overloaded line experiences an outage, its power flow limit $C_{l}^{O P A}$ is divided by a very large number $k_{1}$ to ensure that practically no power may flow through the line. Besides, to avoid a matrix singularity from the line outage, the impedances of failed lines are multiplied by a large number $k_{2}$, resulting in changes of the network matrix $A$.

Load shedding is utilized to quantify the damage of the cascading failure. For an individual node, load shedding is defined as the absolute value of the difference between its power injection and demand:

$$
L S_{j}=\left|P_{j}^{d e m}-P_{j}\right|, j \in V_{D}
$$

Subsequently, total load shedding for the system is:

$$
L S=\sum_{j \in V_{D}} L S_{j}
$$

Finally, system load shedding is normalized by its total demand $\mathrm{D}$ and used as a measure of damage to the system resulting from a cascading failure:

$$
V_{O P A}=\frac{L S}{D}=\frac{\sum_{j \in V_{D}} L S_{j}}{\sum_{j \in V_{D}} P_{j}^{d e m}}
$$

The fact that simulation results from OPA model are consistent with historical blackout data for real power systems has justified its effectiveness [11]. However, the applications of OPA have generally been limited to networks with a relatively small number of nodes compared to real power grids [23], due to the computational efforts involved.

\section{Formulation Of The Multi-ObJective Optimization PROBLEM}

In this section, we generally frame the problem of searching the most favourable pattern of link capacities in a realistic power transmission network, so as to optimize its resilience against cascading failures. By associating a cost to (the capacity of) each link of the network, the optimization process also seeks to minimize the total cost. With the aim of comparing network-centric and power flow approaches, both the ML and OPA models introduced in Section II are used to evaluate the vulnerability of the pattern of link capacities proposed during the optimization search.

Specifically, we define the variables to be optimized as the capacities of the links in the network, $C_{l}, l \in E$ (i.e., $C_{l}^{M L}$ for the ML model and $C_{l}^{O P A}$ for the OPA model). Thus, the homogeneous capacity allocation strategy as expressed in Eq. (2) is no longer adopted in the optimization. Instead, any non-negative vector $C \in \boldsymbol{R}_{+}^{M}$ could represent a potential solution. It is noted that the searching space $\boldsymbol{R}_{+}^{M}$ is intractably large in reality, where a power transmission network usually has hundreds or thousands of links. 
We, then, assume that the cost associated with each link capacity is linearly proportional to the value of the capacity, with coefficient $\varphi$ (we simply set $\varphi$ as 1 in our case study). The total investment cost related to a capacity allocation pattern $C \in \boldsymbol{R}_{+}^{M}$ in the power transmission network can, then, be defined as:

$$
\operatorname{Cost}(C)=\sum_{l \in E} \varphi C_{l}
$$

The network damage resulting from a cascading failure in the presence of a given capacity pattern can be obtained by running the ML (or the OPA) simulation in correspondence of the capacity pattern and, then, using Eq. (3) (or Eq. (9) for OPA). The cascade is initiated by the failure of a single link in each model. The single link is randomly selected from the set of links $E$ in the network with equal probability. Then, the algorithms for cascading simulation proposed in Section II are applied. The cascade simulations run over several iterations until they either converge or exceed the maximum number of steps (we use maximum 20 iterations for both ML and OPA). Finally, the network vulnerability for a given capacity allocation pattern $C$ is obtained as the average network damage $\overline{V_{M L}}$ (or $\overline{V_{O P A}}$ for OPA), over various random triggers (we use 30 triggers for both ML and OPA).

Through the quantification of the capacity allocation cost and cascading failure vulnerability, the capacity allocation problem is formulated as a multi-objective optimization:

$$
\left\{\begin{array}{l}
\min _{C \in R_{+}^{M}} \operatorname{Cost}(C) \\
\min _{C \in R_{+}^{M}} \bar{V}(C)
\end{array}\right.
$$

The objective function (11) is the sum of the link capacity costs; function (12) expresses the cascade vulnerability objective, where $\bar{V}(C)$ is $\overline{V_{M L}}$ when the ML model is used, or $\overline{V_{O P A}}$ when OPA is used. Observe that under this definition the most cascade-resilient network might be the network with infinite capacity, which obviously would conflict with the objective of minimizing cost.

\section{Multi-ObJective Evolutionary Algorithms (MOEA) FOR OPTIMAL CAPACITY ALLOCATION}

Multi-objective evolutionary algorithms (MOEAs) have proven to be general, robust and powerful search tools that are desirable for tackling problems involving i) multiple conflicting objectives, and ii) intractably large and highly complex search spaces [34]. In extreme synthesis, the main properties of Evolutionary Algorithms (EAs) are that the search for the optima is conducted (i) using a (possibly) large population of multiple solution points or candidates, (ii) using operations inspired by the evolution of species, such as breeding and genetic mutation, (iii) using probabilistic operations and (iv) using information on the objective or search functions and not on its derivatives. The main advantages are: (i) fast convergence to near global optima, (ii) superior global searching capability in complicated search spaces and (iii) applicability even when gradient information is not readily achievable. MOEAs rely on the following concepts [35]:

- Pareto front: The locus that is formed by a set of solutions that are equally good when compared to other solutions of that set is called Pareto front.

- Non-Domination: Non-dominated or Pareto-optimal solutions are those solutions in the set which do not dominate each other, i.e., neither of them is better than the other in all the objective function evaluations. The solutions on each Pareto front are Pareto-optimal with respect to each other.

In this study, we use a fast and elitist genetic algorithm, namely, NSGA-II [22], to solve the multi-objective optimization problem (11)-(12). NSGA-II has been proved to be an efficient algorithm to find Pareto optimal solutions [36]; for further details about this algorithm and relevant surveys on multi-objective evolutionary optimization, the reader is referred to Ref. [22], [34]-[36]. The complete procedure for our capacity allocation optimization problem is detailed as follows:

(1) Read power transmission network data (line, bus, adjacency matrix, etc.) and fix the MOEA parameters (i.e., population size, maximum generation, etc.);

(2) Randomly initialize a (parent) population of possible solutions (individuals) and evaluate the fitness of each individual with respect to the two objective functions (11) and (12); sort the parent population according to the non-domination criterion [35];

(3) Select the parents which are fitter for reproduction by using a binary tournament selection [22]; the procedure is such that fitter individuals are selected with a higher probability;

(4) Generate an offspring population by crossover and mutation operators, and evaluate the fitness of each individual in the offspring population with respect to the two objective functions (11) and (12);

(5) Combine the parent and offspring populations to generate a new "trial" aggregate population and perform non-dominated sorting on the "trial" population;

(6) Generate a new parent population by selecting the best solutions in the sorted "trial" population, until a desired population size is reached;

(7) If the stop condition is met, then terminate the iteration; otherwise, go to step 3.

The non-dominated solutions of the last population constitute the Pareto optimal front of the optimization problem at hand. 


\section{CAse Study And Results Analysis}

\section{A. Case Study and Parameters Setting}

In this paper, the $400 \mathrm{kV}$ French power transmission network (FPTN400) (Fig. 1) is taken for exemplification of the proposed approach. The network is built from the data on the $400 \mathrm{kV}$ transmission lines of the RTE website [37]. It has 171 nodes (substations) and 220 edges (transmission lines). We distinguish the generators, which are the source of power, from the other distribution substations, that receive power and transmit it to other substations or distribute it in local distribution grids. By obtaining the power plants list from EDF website [38] and relating them with the ID of the buses in the transmission network, we have 26 generators and 145 distributors. Only the nuclear power plants, hydroelectric plants and thermal power plants whose installed capacities are larger than $1000 \mathrm{MW}$, are considered. Although simplifications have been made, the network model still has sufficient details to illustrate the validity of the method on a realistic-size electrical infrastructure.

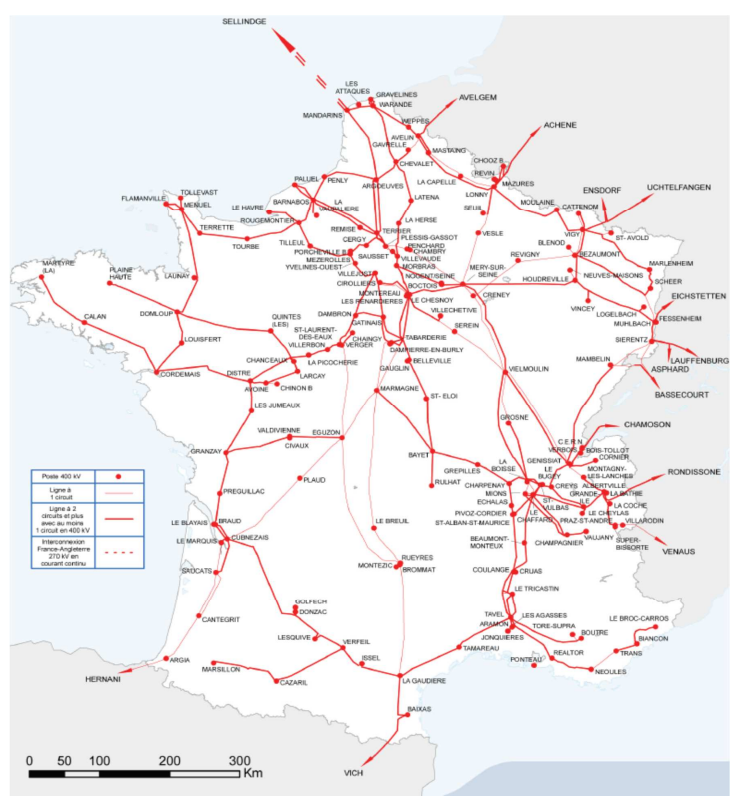

Fig. 1. The 400kV French power transmission network (FPTN400) [37].

For optimal allocation of link capacity in the network, the NSGA-II algorithm introduced in Section IV is applied with regards to the objectives of minimizing cascade vulnerability and investment cost, expressed by functions (11) and (12) respectively. Both the ML and OPA models are used to evaluate the cascade vulnerability of the proposed network. The parameters values used in the NSGA-II algorithm are reported in Table I. In this study, we do not attempt to find the best optimal setting for each of the NSGA-II parameters and they have been set by trial and error guided by the aim of reaching convergence. For the interested reader, extensive studies exist especially focusing on the task of tuning GA parameters [40], [41], [42].
TABLE I

PARAMETERS OF THE NSGA-II ALGORITHM

\begin{tabular}{ll}
\hline \multicolumn{1}{c}{ Parameters } & \multicolumn{2}{c}{ Values } \\
\hline Population size & 80 \\
Maximum generation & 1500 \\
Crossover probability & 0.9 \\
Mutation probability & 0.1 \\
Crossover operator & 20 \\
Mutation operator & 20 \\
\hline \hline
\end{tabular}

\section{B. Comparison between the ML and OPA Models}

\section{1) Model Adjustments and Settings}

The comparison between the optimization results of the ML and OPA models is not straightforward due to the differences of the two models in the way of representing system flow, in the iterative algorithms they rely on, and in the way of measuring the damage produced by the cascading failure. Accordingly, some assumptions and adjustments to the models are necessary to ensure their comparability.

Flow initialization: In the ML model, initial link flow is calculated directly by Eq. (1). Regarding the OPA model, the calculation of initial link power flow by Eq. (5) necessitates data about power demand and generator capacity. Prior studies set this data by evolving the network using combined fast-slow dynamics until the network reaches a steady state [10], [11]. In order to ensure comparability with ML, and taking into account that we limit the scope of our comparison to fast dynamics, we use a simpler initialization strategy that does not require the consideration of network upgrades over time.

Although the ML model does not represent demand and generation capacity quantitatively, it assumes that every distributor is connected to every generator, whereby there is only one shortest path from any distributor to every generator. This implies that every distributor attempts to extract an equal amount of power from every generator [29]. Thus, to facilitate comparability with the ML model, we use the following assumptions in OPA: (i) all the loads have equal constant power demand, and (ii) the total generation capacity is set to be equal to the total demand and equally divided among the generators.

In Fig. 2, we plot the relationship between the initial flow of each link determined using the ML model and that determined using the OPA model in the FPTN400. Each green square in the Figure corresponds to one of the links in the network. The $\mathrm{x}$-axis is the value of initial flow of the link in ML, and its $y$-axis is the value of its initial flow in the OPA approach. It can be seen that the initial link flow in ML is highly correlated with the initial link flow in OPA, computed by means of the proposed initialization method (the correlation coefficient $r_{M L, O P A}$ is equal to 0.77$)$. That is to say, links with high initial flow in ML tend to have high initial flow in OPA, and vice versa. This shows that our initialization strategy is consistent for ML and OPA.

Cost normalization: Since the ML and OPA models rely on different variables and algorithms (see Section II), the numerical values of each link flow and capacity determined within the two approaches are obviously not identical. Therefore, in order to facilitate the comparison of the 
optimization results from the two approaches, the cost of each capacity (allocation pattern) proposed by the optimization algorithm is normalized by the corresponding total initial network flow ${ }^{2}$, and indicated as $\overline{\text { Cost }}$ in both the ML and OPA models.

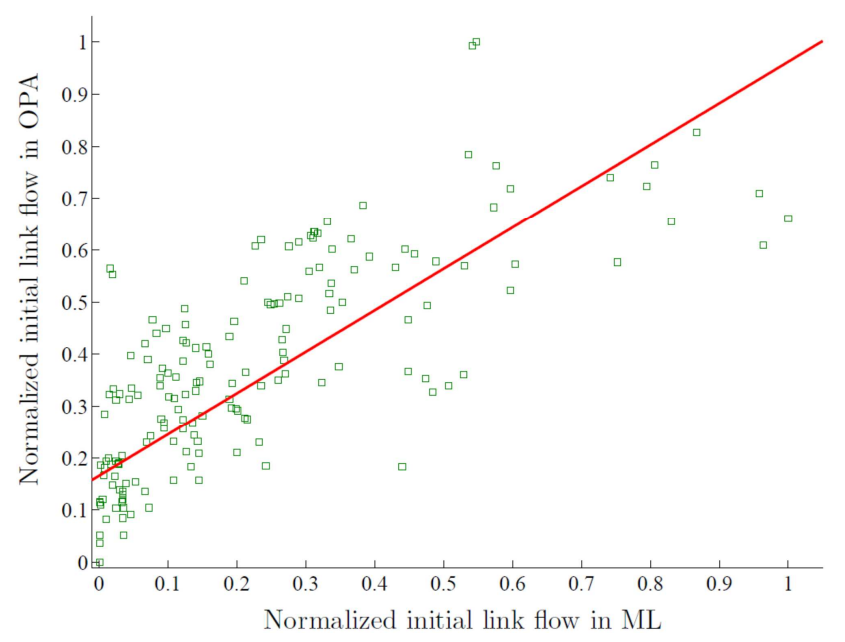

Fig. 2. Scatter-plot of the normalized initial link flows in the ML and OPA models, with reference to the $400 \mathrm{kV}$ French power transmission network. The initial link flow in ML is highly correlated to that in OPA $\left(r_{M L, O P A}=0.77\right)$. The best fit line is also shown.

Comparison method: As already mentioned before, it is evident that the ML and OPA models provide different results at the local scale [29]; however, we evaluate to what extent the two approaches are consistent at the global system level. In particular, we compare the two approaches by performing the following analyses:

- We verify whether the Pareto fronts based on the ML and OPA models exhibit similar characteristics in terms of phase transitions of cascade vulnerability with respect to normalized investment cost;

- We investigate whether the Pareto optimal solutions showing the same level of investment cost also present similar capacity allocation patterns;

- We examine whether the link capacities patterns along the two optimal frontiers exhibit similar characteristics for decreasing network vulnerability (i.e. for increasing network resilience)

\section{2) Comparison Results}

We first investigate the shape of the Pareto fronts obtained using the ML and OPA models in the capacity allocation optimization: in particular, we analyze the variation of cascade vulnerability as a function of normalized investment cost. Notice that a proper comparison of the Pareto fronts obtained with the ML and OPA models is only possible with the adjustments proposed in previous Section. Fig. 3 shows that ML and OPA Pareto fronts exhibit similar phase transitions (although their absolute values are different, which is not unexpected considering the fact that they apply different

\footnotetext{
${ }^{2}$ By this definition, the normalized cost has precisely the same physical meaning with the network tolerance parameter $\alpha$.
}

modelling parameters and cascade vulnerability measures): both curves present a sharp decrease in network vulnerability in the same $\overline{\operatorname{Cost}}$ region (i.e. $1.0 \leq \overline{\operatorname{Cost}} \leq 1.5$ ), where a small increase in the cost gives a large gain in terms of cascade resilience. Besides, regions of plateau exist for certain cost values in both models (i.e. for $1.5 \leq \overline{\operatorname{Cost}} \leq 1.75$ and $2.0 \leq \overline{\operatorname{Cost}} \leq 2.2$ in $\mathrm{ML}$, and for $1.5 \leq \overline{\operatorname{Cos} t} \leq 1.8$ and $2.15 \leq \overline{\operatorname{Cost}} \leq 2.45$ in OPA), in which increasing investment cost does not improve network resilience. Finally, both curves show a relatively stable regime for large $\overline{\operatorname{Cost}}$ values (i.e., $\overline{\text { Cost }} \geq 2.2$ ), where network resilience is already high and its relative improvement is negligible even for a significant increase in the network cost (for example, referring to the ML model, increasing $\overline{\operatorname{Cost}}$ from 1.97 to 2.61 , i.e., of $32.5 \%$, we reduce the network vulnerability of only $1.5 \%$ ). One could refer to the Pareto fronts of ML (squares in left panel) and OPA (triangles in right panel) in Fig. 4, where this relative stable regime is shown more clearly on a linear $y$-axis scale.

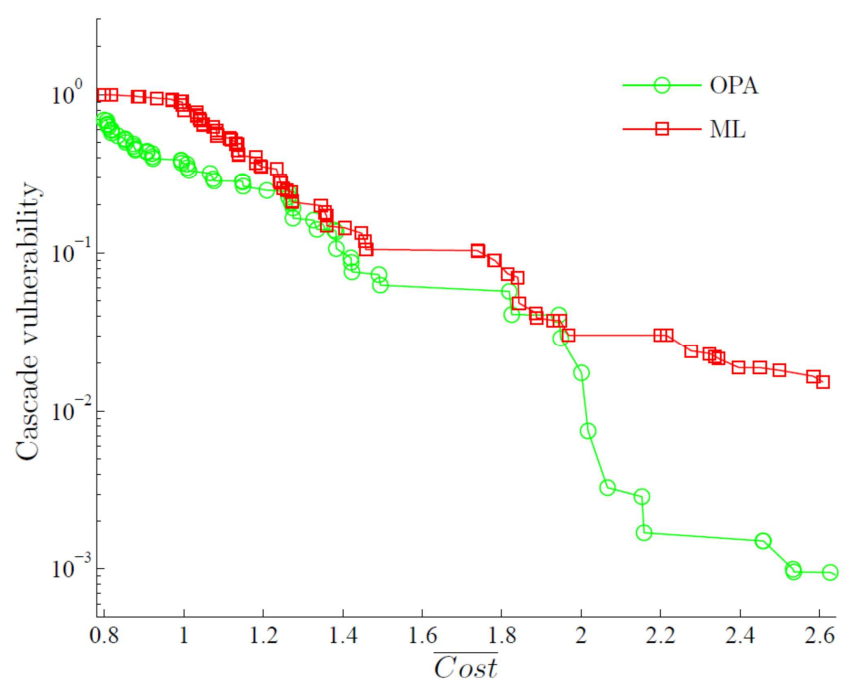

Fig. 3. Phase transitions in the Pareto optimal fronts showing cascade vulnerability (i.e., average efficiency loss for ML and average load shedding for OPA) with respect to normalized investment cost.

In Fig. 4 we compare the Pareto fronts obtained by the ML and OPA models within the multi-objective optimization framework of Section III with the results obtained by assuming a classical homogeneous capacity allocation strategy (see Section II.A). The capacity in the homogeneous capacity allocation is assumed to be linearly proportional to the initial flow by means of the network tolerance parameter $\alpha$, as indicated in Eq. (2); thus, the normalized cost of a given capacity allocation pattern is precisely equal to parameter $\alpha$ by construction. It can be seen that in both cases the multi-objective optimization approach based on ML and OPA produces superior solutions as the corresponding Pareto fronts are closer to the coordinate axes. The linear (homogeneous) capacity-load relationship evidently appears not optimal for obtaining a cost-efficient and cascade-resilient network.

We, then, compare the link capacities patterns of those solutions along the two Pareto fronts that present 

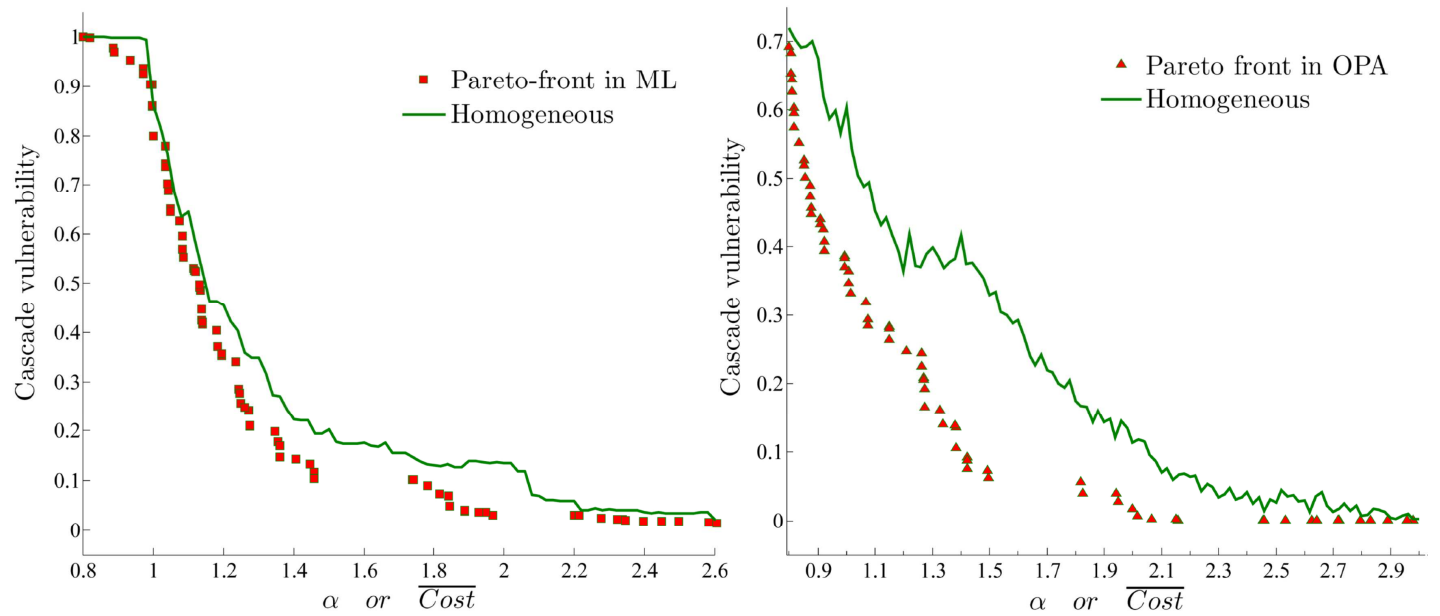

Fig. 4. ML (left panel) and OPA (right panel) Pareto fronts obtained in the multi-objective optimization framework of Section III (squares and triangles), together with the results obtained by employing a homogeneous capacity allocation strategy (solid line).

approximately the same values of $\overline{\operatorname{Cost}}$. In particular, three representative values of normalized cost (i.e., $\overline{\operatorname{Cost}}=1.07,1.27$ and 1.81) along the Pareto fronts are chosen, and the relationship between the link capacities of the corresponding optimal solutions obtained by the ML and OPA models are visualized using the scatterplots of Fig. 5(a), (b) and (c), respectively. It is evident that the link capacities of the optimal solutions based on the ML and OPA models are highly correlated (with correlation coefficient $r_{M L, O P A}=0.73,0.69$ and 0.76 , respectively). That is, links with low capacity in the ML model are likely to have low capacity also in the OPA model, and links with high capacity in ML also have high capacity in OPA.

Finally, it is interesting to analyse how the pattern of link capacities changes when lower network cascade vulnerability (higher network resilience) is demanded, i.e., which type of capacity allocation pattern is the most favourable in resisting to cascading failure. We tackle this problem by investigating the "expected" network link capacity pattern as a function of cascade vulnerability, i.e., the configuration of capacity pattern "averaged" over all possible solutions of the Pareto front lying within a given "regime" (i.e., interval) of cascade vulnerability of interest. Parameter $\beta^{S}$ (namely, $\beta_{M L}^{S}$ for ML and $\beta_{O P A}^{S}$ for OPA) is used to represent the "regime" of vulnerability, where $\mathrm{s}$ indicates the size of the corresponding interval. It is noted that smaller $\beta^{s}$ represents higher network resilience.

Fig. 6 reports the results of averaged link capacities patterns for three different levels of cascade vulnerability, i.e., $0.6 \leq \beta^{0.1} \leq 0.7,0.3 \leq \beta^{0.1} \leq 0.4$ and $0 \leq \beta^{0.1} \leq 0.1$ in the case of a homogeneous allocation strategy (circles) and of the optimization-based approach in our study (squares). The left panel $(\mathrm{a}-\mathrm{c})$ is referred to ML, whereas the right panel (d-f) relates to OPA. It is found that the optimal link capacity patterns exhibit consistent characteristics between ML and OPA models. For example, in both cases, the optimal link capacities patterns are similar to their corresponding homogeneous allocations only in less resilient networks, i.e., when $0.6 \leq \beta^{0.1} \leq 0.7$, where the objective of minimizing investment cost is much more biased (Fig. 6(a) and (d)). When we increase the importance of minimizing the network vulnerability (e.g., for $0.3 \leq \beta^{0.1} \leq 0.4$ and $0 \leq \beta^{0.1} \leq 0.1$ ), the optimal link capacities show a non-linear relationship with respect to their initial flows, as shown in Fig. 6(b), (c) and Fig. 6(e), (f). Specifically, the heavily loaded links tend to decrease their capacities and the lightly loaded links tend to increase their capacities. That is to say, the unoccupied portion of capacity tends to decrease in links with larger loads and the unoccupied portion of capacity tends to increase in the less loaded links. Furthermore, the more importance is given to the minimization of network cascade vulnerability, the more pronounced the non-linear behaviour is, as shown in Fig. 6(c) and (f). Our findings are consistent with the empirical observations and results from the traffic fluctuation model [17], [19].

\section{DISCUSSION AND CONCLUSION}

In this paper, we have tackled the problem of searching for the most favourable pattern of link capacity allocation for a CI network with the objective of resisting to cascading failures with limited investment costs. The problem has been formulated within a multi-objective optimization framework and has been solved by an evolutionary algorithm, namely the NSGA-II. The optimization has been carried out using two different approaches to cascade failure modelling: a computationally-cheap complex network model -- namely, the Motter-Lai (ML) model -- and a more detailed power flow model -- namely, the ORNL-Pserc-Alaska (OPA) model. The approaches have been compared on a case study involving the $400 \mathrm{kV}$ French power transmission network (FPTN400). Although simplifications have been applied, the network model still has sufficient detail to illustrate the validity of the method on a realistic electrical infrastructure.

The objective of this paper is twofold: 1) to tackle the issue of capacity-load relationship from a systematic perspective, by 


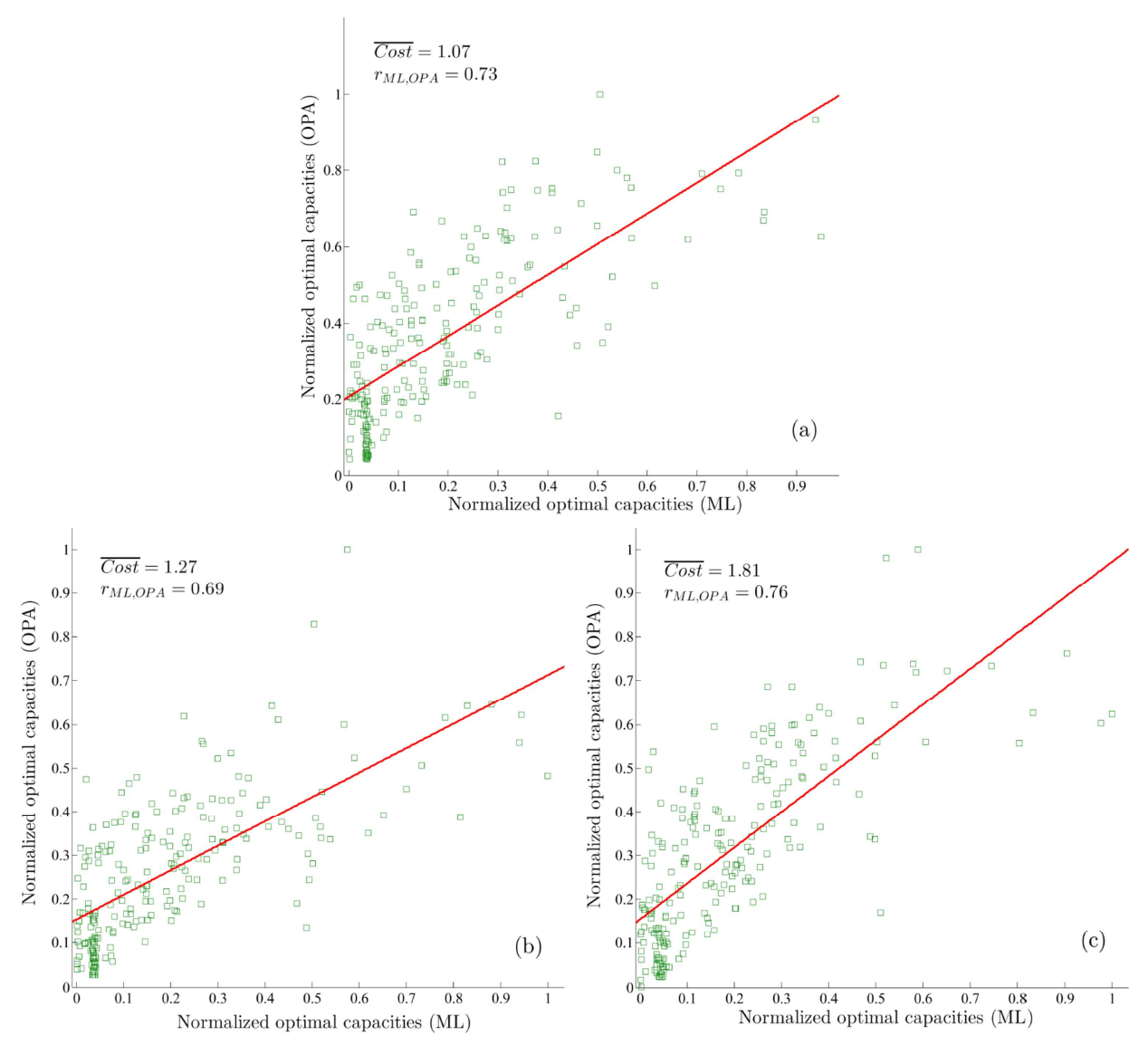

Fig. 5. Scatter plot of the (normalized) link capacities of three representative ML and OPA Pareto solutions showing the same normalized cost. The link capacities of the Pareto solutions with the same level of cost show highly correlated allocation patterns: (a) ML solution (1.07, 0.63) versus OPA solution $(1.07,0.30): r_{M L, O P A}=0.73$; (b) ML solution $(1.27,0.24)$ versus OPA solution $(1.27,0.21): r_{M L, O P A}=0.69$; (c) ML solution $(1.81,0.074)$ versus OPA solution $(1.81,0.057): r_{M L, O P A}=0.76$. The line of best fit is also plotted, for visual guidance.

introducing the optimization of link capacity allocation, and 2) to study the possibility of using a simplified network-centric model (instead of a detailed power flow model) within the optimization framework, without affecting the quality of the optimal solutions found, by embedding both the ML and OPA model into the optimization and comparing their results.

Primarily, our multi-objective optimization results show that both the ML and OPA models produce improved Pareto solutions with respect to those obtained by assuming a classical homogeneous allocation strategy. In addition, the optimal link capacity allocations show a non-linear capacity-load relation: the unoccupied portion of capacity tends to decrease in links with larger loads, whereas the unoccupied portion of capacity tends to increase in the lightly loaded links. This is in sharp contrast with the linear capacity-load relation hypothesized in previous works of literature [8], [9], [12]-[14], [18]. This non-linear behaviour is probably a consequence of the following observation: since larger loads in heavily loaded components tend to result from a large number of flow events, the relative size of the fluctuations in these components tends to be small when other lightly loaded components fail during a cascading failure; considering that the unoccupied capacity is the operating margin that allow safe operation for the component under potential load increment (mainly determined by the perturbations caused by the failure of other components of the network), this explains why in the optimal solutions the unoccupied capacity tends to be smaller for links with larger loads.

Additionally, the analysis of the behaviour of the link capacity patterns of the Pareto optimal solutions as a function of the vulnerability level has shown that the results provided by ML and OPA are consistent: the more importance is given to the objective of network cascade vulnerability, the more pronounced is the non-linear capacity-load relation for both models. Besides, the Pareto fronts produced by ML and OPA exhibit similar phase transitions. Both curves exhibit a sharp decrease in network vulnerability when $1.0 \leq \overline{\operatorname{Cost}} \leq 1.5$, a plateau for certain cost values (i.e., for $1.5 \leq \overline{\operatorname{Cost}} \leq 1.75$ and $2.0 \leq \overline{\operatorname{Cost}} \leq 2.2$ in $\mathrm{ML}$, and for $1.5 \leq \overline{\operatorname{Cos} t} \leq 1.8$ and $2.15 \leq \overline{\operatorname{Cost}} \leq 2.45$ in OPA) and a relatively stable regime when $\overline{\text { Cost }} \geq 2.2$. Furthermore, the link capacities of the Pareto optimal solutions produced by the ML and OPA models show highly correlated allocation pattern, which means that links with low capacity in ML tend to have low capacity in OPA, and links with high capacity in ML also tend to have high 

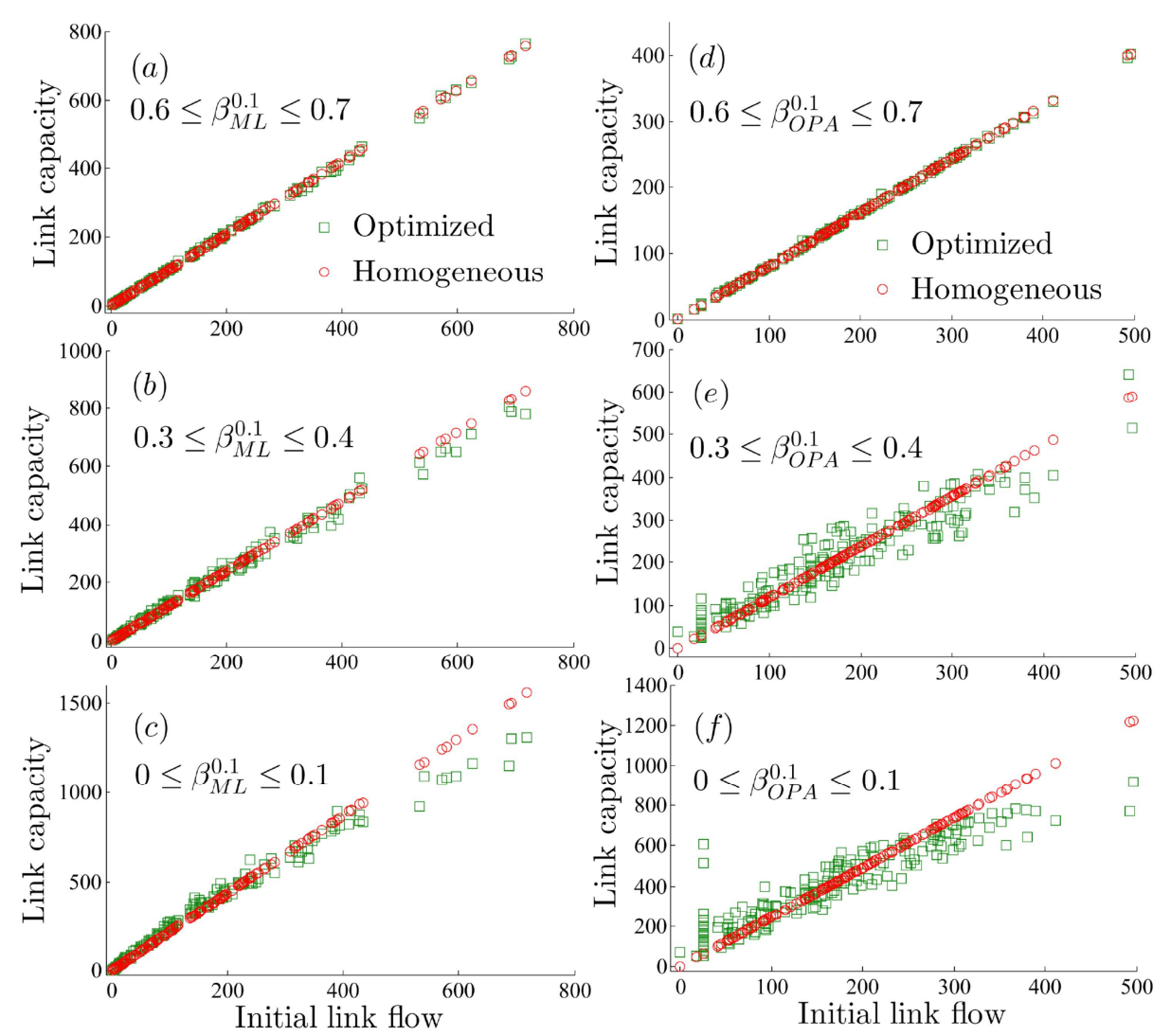

Fig. 6. "Averaged" optimal link capacity patterns for three different levels of cascade vulnerability $\left(0.6 \leq \beta^{0.1} \leq 0.7,0.3 \leq \beta^{0.1} \leq 0.4\right.$ and $0 \leq \beta^{0.1} \leq 0.1$ ) in ML (left panel a-c) and OPA (right panel d-f). The scatter plot shows the relationship between the link capacities and the initial link flows in a homogeneous allocation strategy, where the capacity of a link is assumed to be proportional to its initial flow (circles) and after in the optimization-based approach of Section III (squares).

capacity in OPA. This consistency is not insignificant since it demonstrates that one resilience-improved pattern of capacity allocation optimized by the ML model is also of higher resilience if measured by the more realistic OPA model.

The results from this comparative study provide an important contribution regarding the usefulness of a topological model (ML) in the optimization of a cascade resilient electrical network. Although ML is a relatively simple and abstract model (that does not account for the power flow laws and constraints of the electrical system), it is able to provide results that are consistent with a detailed and more realistic power flow model (OPA), when applied to the problem of network optimization against cascading failure. Most importantly, with respect to OPA it has the advantages of simplicity and scalability: the average time needed to carry out a single cascade failure simulation is $3.9 \mathrm{~s}$ and $20.8 \mathrm{~s}$ for ML and OPA, respectively, on a double $2.4 \mathrm{GHz}$ Intel CPU and 4 GB RAM computer. This provides impetus for the use of network-centric models to the study of cascading failure in large power network systems.

Future works may consider comparing our optimization results with real data, i.e. the empirical capacity-load characteristics, for extracting further insights about how realistic infrastructure systems evolve. Besides, it is noted that the optimization based on the OPA model leads to solutions of reduced vulnerability compared to its ML counterpart (see Fig. 4) and the modelling reason behind it, is worthy of further study. Furthermore, Newton Raphson-based power flow approaches [43] could be applied for the comparison with the ML model, since they give a more detailed depiction of the cascading failure process, although the price to be paid is that they are computationally expensive. Finally, it would be interesting to apply our method to other networks, e.g. the standard IEEE Power Systems Test Cases and the like.

\section{REFERENCES}

[1] U.-C. P. S. O. T. Force, "Final report on the august 14, 2003 blackout in the United States and Canada: causes and recommendations," U.S.-Canada Power System Outage Task Force, Tech. Rep., 2004.

[2] U. for the Coordination of Transmission of Electricity, "Final report system disturbance on 4 nov. 2006," Union for the Coordination of Transmission of Electricity, Tech. Rep., 2007.

[3] J. J. Romero, "Blackouts illuminate India's power problems," Spectrum, IEEE, vol. 49, no. 10, pp. 11-12, 2012.

[4] S. Battiston, D. Delli Gatti, M. Gallegati, B. Greenwald, and J. E. Stiglitz, "Credit chains and bankruptcy propagation in production networks," Journal of Economic Dynamics and Control, vol. 31, no. 6, pp. 20612084, 2007.

[5] M. E. Newman, S. Forrest, and J. Balthrop, "Email networks and the spread of computer viruses," Physical Review E, vol. 66, no. 3, p. 035101 , 2002. 
[6] K. Zhao, A. Kumar, T. P. Harrison, and J. Yen, "Analyzing the resilience of complex supply network topologies against random and targeted disruptions," Systems Journal, IEEE, vol. 5, no. 1, pp. 28-39, 2011.

[7] D. Kempe, J. Kleinberg, and 'E. Tardos, "Maximizing the spread of influence through a social network," in Proceedings of the $9^{\text {th }} A C M$ SIGKDD international conference on Knowledge discovery and data mining. ACM, 2003, pp. 137-146.

[8] A. E. Motter and Y.-C. Lai, "Cascade-based attacks on complex networks," Physical Review E, vol. 66, no. 6, p. 065102, 2002.

[9] P. Crucitti, V. Latora, and M. Marchiori, "Model for cascading failures in complex networks," Physical Review E, vol. 69, no. 4, p. 045104, 2004.

[10] I. Dobson, B. A. Carreras, V. E. Lynch, and D. E. Newman, "An initia model for complex dynamics in electric power system blackouts," in Proceedings of Annual Hawaii International Conference on System Sciences, 2001, pp. 51-51.

[11] B. A. Carreras, D. E. Newman, I. Dobson, and A. B. Poole, "Evidence for self-organized criticality in a time series of electric power system blackouts," Circuits and Systems I: Regular Papers, IEEE Transactions on, vol. 51, no. 9, pp. 1733-1740, 2004.

[12] A. E. Motter, "Cascade control and defense in complex networks," Physical Review Letters, vol. 93, no. 9, p. 098701, 2004.

[13] Y. Li, G. Sansavini, and E. Zio, "Non-dominated sorting binary differential evolution for the multi-objective optimization of cascading failures protection in complex networks," Reliability Engineering \& System Safety, vol. 111, no. 0, pp. 195 - 205, 2013.

[14] Y.-P. Fang, E. Zio et al., "Optimal production facility allocation for failure resilient critical infrastructures," in Proceedings of the $22^{\text {nd }}$ European Safety and Reliability (ESREL 2013) annual conference, Sep. 2013, pp. 2605-2612.

[15] L. Zhao, K. Park, and Y.-C. Lai, "Attack vulnerability of scale-free networks due to cascading breakdown," Physical review E, vol. 70, no. 3, p. 035101, 2004.

[16] R. Kinney, P. Crucitti, R. Albert, and V. Latora, "Modeling cascading failures in the north American power grid," The European Physical Journal B - Condensed Matter and Complex Systems, vol. 46, no. 1, pp. 101-107, 2005.

[17] D.-H. Kim and A. E. Motter, "Fluctuation-driven capacity distribution in complex networks," New Journal of Physics, vol. 10, no. 5, p. 053022, 2008 .

[18] E. Zio and G. Sansavini, "Modeling interdependent network systems for identifying cascade-safe operating margins," Reliability, IEEE Transactions on, vol. 60, no. 1, pp. 94-101, 2011.

[19] D.-H. Kim and A. E. Motter, "Resource allocation pattern in infrastructure networks," Journal of physics A: mathematical and theoretical, vol. 41, no. 22, p. 224019, 2008.

[20] B. Wang and B. J. Kim, "A high-robustness and low-cost model for cascading failures," EPL (Europhysics Letters), vol. 78, no. 4, p. 48001 2007.

[21] P. Li, B.-H. Wang, H. Sun, P. Gao, and T. Zhou, "A limited resource model of fault-tolerant capability against cascading failure of complex network," The European Physical Journal B, vol. 62, no. 1, pp. 101-104, 2008.

[22] K. Deb, A. Pratap, S. Agarwal, and T. Meyarivan, "A fast and elitist multiobjective genetic algorithm: Nsga-ii," Evolutionary Computation, IEEE Transactions on, vol. 6, no. 2, pp. 182-197, 2002

[23] K. Sun and Z.-X. Han, "Analysis and comparison on several kinds of models of cascading failure in power system," in Transmission and Distribution Conference and Exhibition: Asia and Pacific, 2005 IEEE/PES. IEEE, 2005, pp. 1-7.

[24] B. Y. Calida, A. V. Gheorghe, R. Unal, and D. Vamanu, "Dealing with next generation infrastructures academic programmes complexity induced resiliency assessment," International Journal of Critical Infrastructures, vol. 6, no. 4, pp. 347-362, 2010.

[25] S. LaRocca, J. Johansson, H. Hassel, and S. Guikema, "Topological performance measures as surrogates for physical flow models for risk and vulnerability analysis for electric power systems," arXiv preprint, arXiv:1306.6696, 2013

[26] P. Zhang, B. Cheng, Z. Zhao, D. Li, G. Lu, Y. Wang, and J. Xiao, "The robustness of interdependent transportation networks under targeted attack," EPL (Europhysics Letters), vol. 103, no. 6, p. 68005, 2013.

[27] R. Baldick, B. Chowdhury, I. Dobson, Z. Dong, B. Gou, D. Hawkins, H. Huang, M. Joung, D. Kirschen, F. Li et al., "Initial review of methods for cascading failure analysis in electric power transmission systems IEEE PES CAMS task force on understanding, prediction, mitigation and restoration of cascading failures," in Power and Energy Society General Meeting-Conversion and Delivery of Electrical Energy in the $21^{\text {st }}$ Century, 2008 IEEE. IEEE, 2008, pp. 1-8.

[28] G. J. Correa and J. M. Yusta, "Grid vulnerability analysis based on scale-free graphs versus power flow models," Electric Power Systems Research, vol. 101, pp. 71-79, 2013.

[29] V. Cupac, J. T. Lizier, and M. Prokopenko, "Comparing dynamics of cascading failures between network-centric and power flow models," International Journal of Electrical Power \& Energy Systems, vol. 49, pp. 369-379, 2013.

[30] $\mathrm{A}^{\circ}$. J. Holmgren, "Using graph models to analyze the vulnerability of electric power networks," Risk analysis, vol. 26, no. 4, pp. 955-969, 2006.

[31] K. Purchala, L. Meeus, D. Van Dommelen, and R. Belmans, "Usefulness of dc power flow for active power flow analysis," in Power Engineering Society General Meeting, 2005. IEEE. IEEE, 2005, pp. 454-459.

[32] R. W. Floyd, “Algorithm 97: Shortest path," Commun. ACM, vol. 5, no. 6, pp. 345-, Jun. 1962.

[33] B. P. Flannery, W. H. Press, S. A. Teukolsky, and W. Vetterling, Numerical recipes in $c$, Press Syndicate of the University of Cambridge, New York, 1992.

[34] E. Zitzler, M. Laumanns, and S. Bleuler, "A tutorial on evolutionary multiobjective optimization," in Metaheuristics for Multiobjective Optimisation. Springer, 2004, pp. 3-37.

[35] K. Deb, Multi-objective optimization using evolutionary algorithms, vol. 2012. Chichester: John Wiley \& Sons, 2001.

[36] A. Konak, D. W. Coit, and A. E. Smith, "Multi-objective optimization using genetic algorithms: A tutorial," Reliability Engineering \& System Safety, vol. 91, no. 9, pp. 992-1007, 2006.

[37] RTE, "Le réseau de transport d'électricité 400kv," www.rte-france.com/uploads/media/CS4_2013.pdf, November 2013.

[38] EDF, "En direct de nos centrales," http://france.edf.com/france-45634.html/, Avril 2013.

[39] S. Mei, F. He, X. Zhang, S. Wu, and G. Wang, "An improved OPA model and blackout risk assessment," Power Systems, IEEE Transactions on, 24(2), 814-823, 2009

[40] A. E. Eiben, Z. Michalewicz, M. Schoenauer, and J. E. Smith, "Parameter control in evolutionary algorithms," In Parameter setting in evolutionary algorithms, pp. 19-46, Springer Berlin Heidelberg, 2007.

[41] K. De Jong, "Parameter setting in EAs: a 30 year perspective," In Parameter Setting in Evolutionary Algorithms, pp. 1-18. Springer Berlin Heidelberg, 2007.

[42] M. E. Samples, M. J. Byom, and J. M. Daida, "Parameter sweeps for exploring parameter spaces of genetic and evolutionary algorithms," In Parameter Setting in Evolutionary Algorithms, pp. 161-184, Springer Berlin Heidelberg, 2007.

[43] H. Wang, and J. S. Thorp, "Optimal locations for protection system enhancement: a simulation of cascading outages," Power Delivery, IEEE Transactions on, 16(4), pp. 528-533, 2001. 